\title{
Evaluation of a New Chemiluminescence Immunoassay for Diagnosis of SyPHILIS
}

\author{
Xiaohui Mo, Yuelan Jin, Yang Yang, Weizhong Hu, Weiming Gu \\ The Laboratory of Shanghai Skin Diseases and STD Hospital, Shanghai, China
}

\begin{abstract}
Objective: To assess the sensitivity, specificity, and feasibility of a new chemiluminescence immunoassay (CLIA) in the diagnosis of syphilis.

Methods: At first, a retrospective study was conducted, using 135 documented cases of syphilis and 30 potentially interfering samples and 80 normal sera. A prospective study was also performed by testing 2, 071 unselected samples for routine screening for syphilis. CLIA was compared with a nontreponemal test (TRUST) and a treponemal test (TPPA).

Results: There was an agreement of $100 \%$ between CLIA and TPPA in the respective study. The percentage of agreement among the 245 sera tested was $100.0 \%$. Compared with TPPA, the specificity of CLIA was $99.9 \%(1817 / 1819)$, the sensitivity of CLIA was $100.0 \%(244 / 244)$ in the prospective study. CLIA showed $99.5 \%$ agreement with TPPA by testing 2,071 unselected samples. And CLIA seemed to be more sensitive than TPPA in detecting the samples of primary syphilis.

Conclusions: CLIA is easy to perform and the indicator results are objective and unequivocal. It may be suitable for large-scale screening as a treponemal test substituted for TPPA.

Key words: Syphilis, Treponema pallidum, Chemiluminescence Immunoassay, Serology
\end{abstract}

\section{INTRODUCTION}

In recent years, syphilis infection has become a serious problem in China. Syphilis, caused by the spirochete Treponema pallidum subsp. pallidum, is a multistage disease with diverse and wide-ranging manifestations, making laboratory testing a very important aspect of diagnosis. The traditional approach to syphilis testing involves screening with a nontreponemal test, such as the Toluidine red unheated serum test (TRUST), followed by a confirmatory treponemal test with specificity for treponemal antigens. The Treponema Pallidum Particle Agglutinantion (TPPA) or the Treponema pallidum haemagglutination (TPHA) are commonly used to confirm the reactive results of the nontreponemal screening tests for syphilis in China. Although TPPA and TPHA are considered confirmatory assays with high specificity and sensitivity, the subjective interpretation of weak reactions may lead to bias by improper training of technologists and poor quality of reagents. In addition, false-negative results have been reported in HIV infection and biological false positive results have been described in association with false reactivity in a variety of diseases, such as autoimmune diseases, mixed connective tissue diseases, Leprosy and so on [1-6]. The purpose of the present study was to evaluate a new chemiluminescence immunoassay (CLIA) with recombinant specific treponemal antigens, and the results are compared with TPPA and TRUST. Special emphasis was placed on testing clinically defined specimens, biological false-positives samples and high-risk-patient samples.

\section{SubJECTS AND METHODS}

\section{REAGENTS}

TRUST (Rongsheng, Shanghai, China) was used in nontreponemal tests, which was a macroscopic flocculation card test. TRUST antigen suspension was mixed with the patient's specimen on a white card. TRUST measured antibodies to lipoidal material. If antibodies were present, they were combined with particles and red clumps would be seen on the white cards.

TPPA (Furjirebio, Tokyo, Japan) kit was manufactured using gelatin particle carriers sensitized with purified pathogenic Treponema pallidum (Nichols Strain). Serum containing antibodies to treponemes can react with the sensitized gel particles to form a definite large ring with a rough multiform outer margin of a peripheral agglutination. If antibodies were not present, the particles settled to the bottom of the microtiter tray well and into a compact button. If particles concentrated in the shape of a compact ring with a smooth round outer margin, it was regard as an indeterminate result.

CLIA (Chemclin, Beijing, China) for T. pallidumspecific assay was a one-step sandwich performed. 96well plastic plates were coated with recombinant specific treponemal antigens ( TpN15, TpN17, TpN47). A patient's serum and an isoluminol-antigen conjugate were mixed together and incubated. After one-hour incubation, theses plates were washed and chemiluminescent substrates were added into wells. The light signals were measured by a photomultiplier as relative light units (RLU). The results were evaluated using a cutoff value, which was defined as the mean RLU of negative control samples plus 2.1 times. If its value 
was above the cutoff value $(\mathrm{S} / \mathrm{CO}$ value $>1)$, the result was considered positive while interpreted as negative.

\section{SUBJECTS}

For the retrospective study, the first group sera were collected from Shanghai Skin Disease and STD Hospital in 2007. Samples were stored at -20 or $-70{ }^{\circ} \mathrm{C}$ before analysis. Among them, 135 specimens were diagnosed as syphilis including primary (35 specimens), secondary (40 specimens), latent (20 specimens) and treated syphilis (40 specimens), 30 specimens were of biological false positive reactivity (BFP) in the nontreponemal tests, and 80 specimens were normal healthy sera.

For the prospective study, the second group of 2, 071 samples of clinic outpatients were collected randomly from March 2008 to June 2008. These subjects had syphilis (including primary, secondary, latent and treated syphilis), HIV/AIDS, gonorrhea, NGU, genitals herpes, condyloma acuminate (CA) and other diseases.

\section{Methods of Tests}

The study was performed in two phases, designed to determine the sensitivity and specificity of CLIA. The first phase was a retrospective study. The first group sera were examined by CLIA. All of the sera were previously tested by the TPHA and TRUST. In the second phase of the study, the second group samples were examined by TRUST and TPPA as routine tests. These samples were tested by CLIA at the same time.

In this study, the sample was retested if there were discrepant results among TPPA and CLIA. If there still are discrepant results between TPPA and CLIA, a second serum sample collected at least 2 weeks after the first sample should be requested. Testing of this sample will determine whether the initial result was due to low levels of treponemal antibody (repeat test is reactive) or was a false-positive result (repeat test is nonreactive). In addition, our laboratory had participated in the External Quality Assessment (EQA) of National Center for Clinical Laboratory (NCCL) for Clinical Laboratory. According to the feedback and comments from the EQA laboratory, the percent accuracy of TPPA and TRUST in our laboratory had been identified to be $100 \%$.

\section{RESULTS}

\section{RETROSPECTIVE EXPERIMENT}

A total of 245 serum specimens with known serological status were tested. The finding for the retrospective study is shown in Table 1 . The sensitivity of CLIA based on the 135 serum samples from patients with different stages of syphilis. The sensitivity of CLIA was based on the 135 serum samples from patients with different stages of syphilis, with the percentage of agreement 100\% (135/135). And the specificity was based on the 110 serum samples from the normal sera group and the 30 samples from the BFP group in the nontreponemal tests. The specificity of CLIA was $100 \%(110 / 110)$. The S/CO of the positive samples ranged from 3.40 to 55.31 and most of them were above 5.0 and the ratios of the negative samples ranged from 0.01 to 0.41 .

\section{Prospective ExPeriment}

A prospective study was also performed by testing 2071 samples form collected from STD clinics for routine screening for syphilis. According to the clinic symptoms and laboratory tests, these samples were classified into several groups. All groups of the serological test results are summarized in Table 2. For the syphilis group, the majority of CLIA-positive samples (237 of 245) were apparently TPPA-reactive, and parts of CLIA-positive samples (173 of 245) were TRUSTreactive. In addition, there also are 5 samples diagnosed as positive both in TPPA and CLIA for other groups, four samples from HIV-positive patients, and one sample from CA group. The S/CO value of the positive samples ranged from 1.21 to 57.32 and the results for negative samples ranged from 0.01 to 0.76 . CLIA, calculated by the assay's S/CO value and the sample dilution factors, showed a positive correlation with the titer of TPPA in most of samples. For the positive samples, the assay of S/CO value within the range of 1.21 to 3.45 in CLIA usually was equivalent to a titer of $1: 80$ when tested by TPPA. If the S/CO value of CLIA above this range, Titers of TPPA were predictably above $1: 80$. But there were few exceptions included four latent syphilitic samples and two treated syphilis. The titers of these six cases were above $1: 80$ in TPPA, but the S/CO values were below 3.45 (ranged from 1.37 to 2.45 ) in CLIA.

Table 1. The three tests detected for different groups of sera in respective experiment.

\begin{tabular}{|c|c|c|c|c|c|c|}
\hline \multirow[t]{2}{*}{ Sample population } & \multicolumn{2}{|c|}{$\begin{array}{l}\text { No. of sample with } \\
\text { indicated CLIA result }\end{array}$} & \multicolumn{2}{|c|}{$\begin{array}{l}\text { No. of sample with } \\
\text { indicated TPPA result }\end{array}$} & \multicolumn{2}{|c|}{$\begin{array}{l}\text { No. of sample with } \\
\text { indicated TRUST result }\end{array}$} \\
\hline & Positive & Negative & Positive & Negative & Positive & Negative \\
\hline Primary syphilis & 35 & 0 & 35 & 0 & 30 & 5 \\
\hline Secondary syphilis & 40 & 0 & 40 & 0 & 40 & 0 \\
\hline Latent syphilis & 20 & 0 & 20 & 0 & 10 & 10 \\
\hline Treated syphilis & 40 & 0 & 40 & 0 & 23 & 17 \\
\hline $\mathrm{BFP}$ & 0 & 30 & 0 & 30 & 30 & 0 \\
\hline Normal sera & 0 & 80 & 0 & 80 & 0 & 80 \\
\hline Total & 135 & 110 & 135 & 110 & 133 & 112 \\
\hline
\end{tabular}


Table 2. The three tests detected for different groups of sera in prospective experiment.

\begin{tabular}{|c|c|c|c|c|c|c|c|}
\hline \multirow[t]{2}{*}{ Sample population } & \multicolumn{2}{|c|}{$\begin{array}{l}\text { No. of sample with } \\
\text { indicated CLIA result }\end{array}$} & \multicolumn{3}{|c|}{$\begin{array}{c}\text { No. of sample with indicated } \\
\text { TPPA result }\end{array}$} & \multicolumn{2}{|c|}{$\begin{array}{l}\text { No. of sample with } \\
\text { indicated TRUST result }\end{array}$} \\
\hline & Positive & Negative & Positive & Indeterminate & Negative & Positive & Negative \\
\hline Syphilis & 245 & 0 & 239 & 6 & 0 & 173 & 72 \\
\hline HIV-positive patients & 4 & 6 & 4 & 0 & 6 & 4 & 6 \\
\hline Condyloma acuminate & 1 & 84 & 1 & 0 & 84 & 1 & 84 \\
\hline Hepatitis & 0 & 64 & 0 & 0 & 64 & 0 & 64 \\
\hline Gonorrhea & 0 & 62 & 0 & 0 & 62 & 0 & 62 \\
\hline Other diseases & 0 & 163 & 0 & 0 & 163 & 0 & 163 \\
\hline Routine testing & 2 & 1440 & 0 & 2 & 1440 & 11 & 1431 \\
\hline Total & 252 & 1819 & 244 & 8 & 1819 & 189 & 1882 \\
\hline
\end{tabular}

Table 3. Comparison of CLIA results with TPPA test and TRUST results for 2071 serum samples.

\begin{tabular}{l|lll|ll}
\hline \multirow{2}{*}{ CLIA } & \multicolumn{2}{|c|}{ No. of samples with indicated TPPA result } & \multicolumn{2}{l}{ Comparison with TRUST } \\
& Positive & Equivocal & Negative & Positive & Negative \\
\hline Positive & 244 & 6 & 2 & 178 & 72 \\
Negative & 0 & 2 & 1817 & 11 & 1810 \\
Total & 244 & 8 & 1819 & 189 & 1882 \\
\hline
\end{tabular}

The results of the comparison of CLIA versus TPPA and TRUST for the 2071 serum samples are shown in Table 3. There was an agreement of $99.5 \%$ between CLIA and TPPA. Compared with TPPA, the specificity of CLIA was $99.9 \%(1817 / 1819)$, the sensitivities of CLIA test was $100.0 \%(244 / 244)$. There existed ten of the serum samples not agreeing between CLIA and TPPA. Six indeterminate-TPPA samples in syphilis group were positive in CLIA. Four of them were confirmed as treated syphilis; other two samples of primary syphilis diagnosed by positive findings of silver staining were verified through repetitive tests showing a titer of $1: 80$ in TPPA for the second serum. Two samples from routine screening group were reactive in CLIA, but nonreactive in TPPA. After inquiry, two serum samples were old treated syphilis. Two indeterminate TPPA samples from the routine testing group showed negative in CLIA, which were negative in TPHA (Omega Diagnostics, Alva, UK). Compared with CLIA, TRUST was less sensitive for syphilis $(178 / 252)$. In addition, there were eleven false positive samples in TRUST, whose titers ranged from $1: 1$ to $1: 4$.

\section{PRECISION}

The precision of CLIA was studied using positive and negative controls run once a day. Averages of the intraassay and interassay coefficient of variation $[\mathrm{CV}]$ for the negative control were $5.1 \%$ and $6.5 \%$, respectively; averages of the intraassay and interassay coefficient of variation $[\mathrm{CV}]$ for the positve control were $6.7 \%$ and $8.8 \%$, respectively.

\section{Discussion}

Chemiluminescent immunoassays have been universally performed for the clinic diagnosis in past years, and showed to be at least as sensitive and specific as the traditional techniques such as immunoenzymatic assay $[7,8]$. Our evaluation of CLIA T. pallidum-specific assay were compared with TPPA used in our laboratory. As a new test for routine use in the clinical laboratory, the simplicity with which the test can be performed, and the test's sensitivity and specificity must be considered. TPPA is prone to indeterminate results, exhibiting particles concentrated in the shape of a compact ring with a small smooth round outer margin. Besides, few specimens may cause agglutination with both unsensitized and sensitized particles. Compared with TPPA, the advantages of CLIA are more objective, less labor intensive and automated.

In this study, two different groups of sera were chosen to verify the sensitivity and specificity of CLIA. In respective study, no serum showed a difference between CLIA and TPPA. And CLIA performed with an agreement of $99.5 \%$ and showed a positive correlation with the titer of TPPA for most of samples in the prospective study. While agreeing quite well with TPPA in respective experiment, CLIA seemed to be of higher sensitivity than TPPA in primary syphilis. Two sera from primary syphilis showed indeterminate in TPPA were apparently identical to that determined by CLIA. As specific recombinant antigens of T. pallidum were used in a sandwich manner, which guarantees the specificity of CLIA. False positive or negative results didn't occur in CLIA, while there existed eight equivocal results in TPPA. Therefore, CLIA should be an appropriate alternative to TPPA for use as a confirmatory test, or be used to resolve those equivocal results in TPPA as another treponemal test.

Some samples included four latent and two treated syphilis cases exhibited high titer in TPPA but faint reaction in CLIA. It may be explained that the discordant results due to the different antigens used in dif- 
ferent kits. Some studies described that antigens showed different antibody reactivity at different syphilitic stages [9]. TPPA kit is manufactured using whole pathogenic T. pallidum strain, while CLIA kit used several recombinant antigens of T. pallidum. So CLIA only detected antibodies to these recombinant antigens, but TPPA can detect the antibodies to the whole antigens of $T$. pallidum.

The treponemal tests usually remain positive throughout the patient life and cannot distinguish recent versus remote or previous treated infections. In order to avoid excessive therapy, the consideration of the clinical symptoms should be required and nontreponemal tests such as TRUST or the RPR are commonly used. Quantitative tests allow for the establishment of a baseline titer, which will make possible evaluation of recent infection and response to treatment. This also allows for the detection of reinfection or relapse in patients with a persistently reactive titer. But the nontreponemal tests have a high rate of biological false-positive results. The rate of biological false positive results was nearly $0.53 \%$ in the prospective study and false-positive tests often occurred in samples from the young women.

In a conclusion, CLIA can be used as an automated method for the detection of treponemal antibodies in human serum. With a high sensitivity, it may be suitable for screening the large scale sample following a nontreponemal screening test. If it is placed into routine use in the clinical laboratory, its interpretation must be combined with clinical signs or symptoms and results from other tests to diagnose patients having syphilis or not.

\section{REFERENCES}

1. Noh J, Ko HH, Yun Y. Evaluation of performance and false positivity of Mediace RPR test that uses a chemistry autoanalyzer. Korean J Lab Med. 2008; 28(4):312-8.
2. de Larrañaga G, Trombetta L, Wingeyer SP, Remondino G. False positive reactions in confirmatory tests for syphilis in presence of antiphospholipid antibodies: misdiagnosis with prognostic and social consequences. Dermatol Online J. 2006 May 30;12(4):22.

3. Körber A, Dissemond J, Lehnen MS. Syphilis with HIV coinfection. J Dtsch Dermatol Ges. 2004; 2(10):833-40.

4. Geusau A, Kittler H, Hein U. Biological false-positive tests comprise a high proportion of Venereal Disease Research Laboratory reactions in an analysis of 300,000 sera. Int J STD AIDS. 2005; 16(11):722-6.

5. Marks R, Thomas-Kaskel AK, Schmidt D. Steroid refractory episcleritis as early manifestation of neurosyphilis. Eur J Med Res. 2006 July 31; 11(7):309-12.

6. Ratnam S. The laboratory diagnosis of syphilis. Can J Infect Dis Med Microbiol. 2005; 16(1):45-51.

7. Carrie SK, Mary AC, Robert WH. Evaluation of the LIAISON Chemiluminescence Immunoassay for Diagnosis of Syphils. Clin Vaccine Immunol. 2007; 6:710-3.

8. Antonella M, Vittorio SA, Francesca C. Evaluation of LIAISON Treponema Screen, a Novel Recombinant Antigen-Based Chemmiluminesnce Immunoassay for Laboratory Diagnosis of Syphilis. Clin Diagn Lab Immunol. 2005; 12:1231-4.

9. Brinkman MB, McKevitt M, McLoughlin M, Perez C, Howell J, Weinstock GM, Norris SJ, Palzkill T. Reactivity of antibodies from syphilis patients to a protein array representing the treponema pallidum proteome. J Clin Microbiol. 2006 Mar;44(3):888-91.

Received: November 24, 2008 / Accepted: May 8, 2009

Address for correspondence:

WeiMing Gu

The Laboratory of Shanghai Skin Diseases

and STD Hospital

Shanghai

China

Tel.: $\quad+86-21 / 62522435$

E-mail: weiming_gu2003@yahoo.com.cn 